Editorial

\title{
Editorial - Patient with Recurrent Glioblastoma Responding Favorably to Ketogenic Diet Combined with Intranasal Delivery of Perillyl Alcohol
}

\author{
Editorial - Paciente com glioblastoma recorrente respondendo \\ positivamente a dieta cetogênica combinada a álcool perílico intranasal
}

\author{
Helder Picarelli, MD, $\mathrm{PhD}^{1}$ \\ ${ }^{1}$ Division of Neurosurgery, Instituto do Câncer do Estado de São Paulo \\ Octavio Frias de Oliveira (Icesp), São Paulo, SP, Brazil
}

Arq Bras Neurocir 2017;36:143-144.

Glioblastoma (GBM) is by far the most frequent and malignant type of primary brain tumor in the adult population. Despite considerable efforts and extensive researches, it still has one of the poorest prognoses in cancer diseases. According to the new (2016) World Health Organization (WHO) classification, GBMs are categorized by their histologic findings and diagnostic biomarkers as: a) isocitrate dehydrogenase (IDH) mutant; b) IDH wild type; and c) NOS GBM (IDH1/2 not tested or inconclusive). The majority of GBMs is IDH wild type (90\%), and they occur preferentially in elderly people without a pre-existing lower-grade glioma precursor. Currently, it is believed that the knowledge of the genetic, epigenetic and chromosomal aberrations on GBMs can predict the therapy response and the outcomes. Normally, IDH mutant GBM has IDH1/2, TP53, ATRX mutation, and they are glioma- $\mathrm{CpG}$ island methylator phenotype (G-CIMP). On the other hand, IDH wild-type GBM has TERT, PTEN, TP53, PIK3CA, PIK3R1, NF1, H3F3A mutation; EGFR, PDGRA, MET, CDK4, CDK6, MDM2, MDM4 amplification; EGFRvIII deletion mutation, and they have MGMT promoter methylation. Regardless of the significant changes and refinement of the WHO classification, the treatments and outcomes are still dismal and disappointing, because the patients have a short survival rate and severe disabilities. In order to change this picture, novel treatment strategies are needed.

The extent of the resection is still considered one of the most important positive prognostic factors, but the advances in surgery (such as neuronavigation, monitoring, brain mapping and dyes) appear to have nearly reached their limits in achieving the maximal safe resection. Similarly, the role and the way of performing radiotherapy already seem to be well established. Despite this, they are performed focally (saving critical and functional areas), making it almost impossible to eradicate all cancerous cells, since GBMs are diffuse or multifocal. Eventually, the disease will progress, leading to serious disabilities and low quality of life, unless another effective therapy is performed simultaneously. Although the current adjuvant chemotherapy is only minimally effective in the treatment of GBMs, the addition of temozolomide (an alkylating agent) prolongs the progression-free survival time, especially in the MGMT promoter methylation phenotype tumor. Another concern is that GBM is a very heterogeneous disease, and its cells can promptly acquire new mutations and convert into a therapy-resistant malignancy. In spite of that, many weak key points involved in the growth and apoptosis of cancerouscells (immunomodulatory, metabolic or genetic pathways) could be attacked.

Even though the treatment for GBM recurrence has many ethical and financial implications, the rescue surgery and bevacizumab (humanized monoclonal antibody targeting VEGF) administration seem to be reasonable for patients with good functional status. Recently, many pre-clinical studies and phase II/III clinical trials have been conducted to assess new options. Tumor-treating fields (TTFs) and re-irradiation have been mentioned as promising approaches, but current data also indicates that an effective strategy needs to be a combinatorial and individual therapy according to the tumor's genetic signature. In fact, the target therapy must be the future, and the main approaches in focus are: a) targeting immunosuppressive checkpoints, such as ipilimumab and nivolumab; b) active immunotherapy, such as cancer vaccines and immune stimulatory gene therapy; c) passive immunotherapies using
Address for correspondence Helder Picarelli, MD, PhD, Divisão de Neurocirurgia, Instituto do Câncer do Estado de São Paulo Octavio Frias de Oliveira (Icesp), São Paulo, SP, Brazil (e-mail: hpicarelli@gmail.com; helderpicarelli@gmail.com).
DOI https://doi.org/ 10.1055/s-0037-1606299. ISSN 0103-5355.
Copyright @ 2017 by Thieme Revinter Publicações Ltda, Rio de Janeiro, Brazil

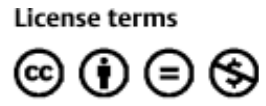


antibodies; and d) adoptive strategies, such as the ones using chimeric antigen receptor (CAR) T cells.

In this issue, Brazilian Neurosurgery publishes a case report of a recurrent GBM patient who was successfully treated combining daily intranasal delivery of monoterpene perillyl alcohol (a Ras/MAPK pathway inhibitor) and a ketogenic diet. The therapeutic potential of this natural, apparently safe and low-cost target therapy for recurrence should not be neglected despite the need for further studies. 